\title{
DIE MARIA-THERESIANISCHE URBARIALVERORDNUNG IM KOMITAT SOPRON/ÖDENBURG
}

\author{
ERNÖ DEÁK \\ Ehemals Österreichische Akademie der Wissenschaften \\ becsi-naplo@aon.at
}

\begin{abstract}
Die Maria Theresianische Urbarialverordnung führte im Wesentlichen, vornehmlich in Hinblick auf die Abgaben und Leistungen keine grundlegenden Änderungen ein, schuf aber in den mitunter unübersichtlichen Zuständen Ordnung und führte landesweit ein einheitliches System ein. Neben etlichen Erleichterungen waren dies Schritte, die den Weg in die Zukunft wiesen.
\end{abstract}

Schlüsselwörter: Reform, Bauernfrage, Urbarialverhältnisse, Robot, Grundherren, Landbesitz

Maria Theresia war eine „Reformerin wider Willen“, meint Martin Mutschlechner über die Regentin. Sie hatte einen durch und durch pragmatischen Zugang zu den Reformmaßnahmen. Nicht die Verfolgung eines philosophischen Gedankens, sondern die praktische Anwendbarkeit stand im Vordergrund. Sie bediente sich zwar einiger Ideen der Aufklärung, aber das ideelle Konzept dieser Strömung als solches blieb ihr fremd. ${ }^{1}$

Die Maßnahmen, die mit dem Namen Maria Theresias verbunden sind, können von ihren Ratgebern, Gerard Van Swieten (Leiden, 1700 - Wien, 1772), Josef von Sonnenfels (Nikolsburg, 1732 - Wien, 1817) und Johann Christoph von Bartenstein (Straßburg, 1689 -Wien, 1776) nicht getrennt werden. Etwas scharf formuliert, gab Maria Theresia den Reformen „lediglich“ ihre Einwilligung und ihren Namen, das eigentliche Reformwerk entwarfen und führten die genannten Persönlichkeiten unter Mitwirkung der Beamtenschaft höheren und höchsten Grades aus. Ohne auf den persönlichen Lebenslauf der Herrscherin eingehen zu wollen, erliegt man leicht der Versuchung, die eigentliche Bedeutung der Reformerin abzutun, was mit ihrem Sohn, Joseph II. in Zusammenhang gebracht werden kann. Denn er soll der eigentliche Reformer gewesen sein, werden doch seine Taten unter dem Sammelbegriff Josephinismus behandelt, während seine Mutter anscheinend nur als eine „Reformerin wider Willen“ genannt wird. Der 300. Geburtstag der Herrscherin könnte dazu dienen, ihre Gestalt auch ohne Huldigung als eine der bedeutendsten Gestalterinnen des Habsburgerreiches 
wiederzuerkennen und anzuerkennen. Selbstverständlich ohne Bezug darauf, dass sie eine Frau war.

Es gilt als Gemeinplatz, die Frevel und Fehler der Regenten auf ihre Ratgeber abzuwälzen, umgekehrt kann es einem - wie eben Maria Theresia und speziell auf ihr Reformwerk bezogen - ergehen, dass dieses Werk vor allem ihren Ratgebern zugeschrieben wird. Diese Problematik bedürfte einer näher eingehenden Behandlung, doch soll dieser Beitrag nicht durch analytische Erwägungen belastet werden. Statt dessen sollen die namentlich genannten Persönlichkeiten etwa mit folgenden Hinweisen bedacht werden: Alle drei genannten Ratgeber waren Migranten und ihr Aufstieg am Wiener Hof war - neben ihren Fähigkeiten Maria Theresia zu verdanken, obwohl sie sich auch in ihren diesbezüglichen Entscheidungen sicherlich beraten ließ. Darüber hinaus ist eine gewisse Reziprozität nicht zu leugnen: Sonnenfels war getaufter Jude und auch Bartenstein konvertierte vom evangelischen zum katholischen Glauben, zudem war er bürgerlicher Herkunft. Die Herrscherin hatte offensichtlich „Augen dafür“, richtige Auswahlen zu treffen, und für die „Auserwählten“ war der dafür nötige Preis nicht unerfüllbar hoch, um der Verlockung nachzugeben.

Bevor nun auf das eigentliche Thema dieses Referates eingegangen wird, soll die Frage beantwortet werden, welche Art von Reformen es waren, die mit dem Namen Maria Theresias verknüpft sind: Sie können unter den Sammelbegriffen Staatsorganisation - Heeresreform - Justiz - Bildungswesen - Bevölkerungspolitik - Religionspolitik - Wirtschaftspolitik zusammengefasst werden. Vornehmlich die letztere, einschließlich der Bevölkerungspolitik stand im Zeichen des Merkantilismus; dieser diente zur Stärkung des Staates, dessen eine der wichtigsten Stützen das stehende Heer war. Allein schon diese Aufzählung lässt eine Verkettung und Vernetzung, mithin Aspekte des angehenden modernen Staates erkennen.

Die wirtschaftlichen Maßnahmen wurden in den Fünfzigerjahren ergriffen und betrafen die Wareneinfuhr bzw. -Ausfuhr. Eine Schutzpolitik also, die zur Erstarkung der heimischen Wirtschaft diente, d.h. die Ausfuhr wurde durch niedrige Zölle erleichtert, die Einfuhr ausländischer Waren mit hohen Zöllen belegt. Dies ging mitunter so weit, dass beispielsweise 1759 die Einfuhr von Metallwaren aus Nürnberg untersagt wurde. Diese Zollpolitik wurde bis zur Schaffung der Zollunion innerhalb der habsburgischen Erbländer forciert (1775). Da das Königreich Ungarn nicht zu diesen gezählt wurde, betraf diese Verordnung Ungarn nicht, was sich auf das Land nachteilig ausgewirkt hatte. Die Einteilung der einzelnen Länder nach wirtschaftlichen Gesichtspunkten bewirkte eine Art Spezialisierung, z.B. sollte in Böhmen die Industrie gefördert werden, in Ungarn dafür die Landwirtschaft dominant bleiben. Die Anfänge dieser Benachteiligung Ungarns gehen auf das Jahr 1755 zurück, in dem die Einfuhr ausländischer Waren durch ungar- 
ländische Kaufleute mit 30\% Zoll belegt wurden. Diese Vorgangsweise wurde allerdings 1773 rückgängig gemacht. ${ }^{2}$

Um so mehr Aufmerksamkeit widmete die Königin in Ungarn der so genannten Bauernfrage. Dies hatte mittelbare und unmittelbare Gründe. Mittelbar war es das Bevölkerungswachstum und die nötige Sicherung der Ernährung und Schaffung neuer Einnahmequellen. Der unmittelbare Grund war jedoch die regional unerträgliche Lage der bäuerlichen Untertanen. Verschiedene Unruhen waren ausgebrochen, wie 1756 in Slavonien (Komitate Szerém, Verőce, Pozsega), sodass die Beruhigung der Lage einer umfassenden Regelung der Urbarialverhältnisse bedurfte. Die eingeleiteten Schritte stießen auf keinen nennenswerten Widerstand seitens des Adels. Eine gewisse „Ermunterung“ griff um sich, und speziell in den westungarischen Komitaten Vas/Eisenburg und Zala fühlten sich die Bauern ermutigt, ihren Beschwerden freie Luft zu machen. Sie wandten sich mit ihrem Anliegen 1765 unmittelbar an die Königin in Wien, zugleich erbaten sie von ihr Schutz und Hilfe gegen die Übergriffe der adeligen Grundherren. Religiosität und königliche Gnade, vor allem aber die Staatsinteressen waren dafür ausschlaggebend, dass Maria Theresia für ganz Ungarn (allerdings ohne Siebenbürgen und Kroatien bzw. das Banat, beide letztere kamen erst 1780 an die Reihe) zunächst eine breit angelegte Untersuchung der Lage auf dem Wege von Befragungen der Bauern verordnete. Diese wurden von Komitatsbeamten unter Aufsicht von königlichen Kommissären durchgeführt. Abgesehen von den ungelösten Fragen, die diese Befragungen aufdeckten, sind die angefertigten und versiegelten Schriften die wichtigsten Quellen ihrer Zeit über die Zustände im Lande. Die Dokumente wurden in drei Sprachen (Lateinisch, Ungarisch, Deutsch), in vier Exemplaren angefertigt und mit dem Siegel des jeweiligen Komitats versehen; je ein Exemplar erhielten der zuständige Grundherr, die Ortsgemeinde, das Komitatsarchiv und die königliche Statthalterei. Dies an sich ein Novum, das - zwar erst 1774 abgeschlossen - allgemeine Gültigkeit erlangte. Die Verhandlungen über die Bauernbefreiung von 1848, die seit 1832 am Landtag als akutes Problem permanent auf der Tagesordnung standen, konnten allenfalls auf diese Dokumente zurückgreifen. Trotz ihrer Brisanz wurden diese Materialien, d.h. die Antworten auf die neun gestellten Fragen bis in die Sechzigerjahre des 20. Jahrhunderts nicht verlegt. Abgesehen von der Edition in einigen Komitaten seit 1967, kann somit die die westungarischen Komitate (Transdanubien) umfassende, groß angelegte Herausgabe des Werkes über die urbarialen Besitzverhätnisse zur Zeit Maria Theresias als erstes grundlegendes Unterfangen betrachtet werden. ${ }^{3}$ Der Initiative der Komitate Pest, Szabolcs, Szatmár, Bereg, Zemplén folgend, traten ungarische und österreichische Historiker auf den Plan und veröffentlichten die dokumentierten Antworten - betreffend das historische Gebiet des Komitates Sopron/Ödenburg, folglich samt Burgenland in zwei Bänden und in drei Sprachen. ${ }^{4}$ 
Der erste Teil erstreckt sich über 98, der zweite Teil auf 108 Gemeinden in ungarischer und lateinischen Sprache, der zweite Teil ist mit Ausnahme einer Gemeinde (Osli) deutsch verfasst. Die Zahl der behandelten Orte ist nicht vollständig, außer einigen Untertanendörfern fehlen die 46 kleinadeligen Gemeinden, die als eigene Grundherren naturgemäß weder zins-, noch robotpflichtig waren. Die Wichtigkeit dieser Bekenntnisse (novem Puncta) veranlasst dazu, sie im Folgenden im damaligen Sprachgebrauch wiederzugeben.

I. Ob und was vor ein Urbarium anjetzo verhanden und was Zeit an selbes errichtet worden?

II. Weill kein Urbarium verhanden ist, werden die Robothen und andere Gaaben vermög Contract oder nur nach eingekommenen Gebrauch von Unterthanen erfordert und was Zeit her ist solcher Contract oder Gebrauch einkommen?

III. Wo keine Urbarien und Contracten vorhanden, in was die herrschaftlichen gewöhnlichen Praestierungen bestehen, wann und mit was für eine Art selbe eingeführet worden?

IV Was dieser Orth insonderheit vor ein Nutzen oder Schaden hat?

V. Mit was und wieviel Joch Äcker und Wüsen ein jeder ganzer Lehner versehen und wieviel Preßburger Metzen in eine jede Joch gehe, ob und auf welchen Wisen auch Grämeth kann gemähet werden?

VI. Was vor Robath, wieviel Täg und mit wieviel Stuk Vieh ein jeder ganzer Lehner zu verrichten hat und ob der Hin- und Rückgang in die Robath gerechnet worden?

VII. Ob das Neuntl von was Zeit und was Gattung gegeben worden und ob das Neuntl auch in anderen Herrschaften gebräuchlich seie und was die Unterthanen den Grundherrn bis auf diese Zeit an anderen Gaaben, Geschenknußen, baaren Geld oder Naturalien specifice gereicht haben?

VIII. Wieviel sich in diesen Orth öede Lehen Häuser befinden, von was vor einer Zeit an und was Ursach und von wem selbe anjetzo genossen werden?

IX. Ob die Unterthanen auf ewig oder auf eine Zeit verpflichtet seind?

Aus den Antworten ergibt sich ein umfassendes, zugleich aber auch differenziertes Bild über die einzelnen Gemeinden, d.h., sie wurden nicht schematisch erfasst, sondern es wurden gleichermaßen die örtlichen Verhältnisse berücksichtigt. Das allerwichtigste war die Klassifikation von Grund und Boden: Je nach Qualität wurden die Äcker und Wiesen in fünf Klassen eingeteilt. Die Äcker wurden nach Joch, die Wiesen nach Tagwerk gerechnet. Da das Joch unterschiedliche Größen zwischen 1.100 und 1.300 bzw. in Slavonien sogar 2.000 Quadratklafter maß, wurde der Einheitlichkeit halber der „Preßburger Metzen“ als Richtmaß 
eingeführt. Demnach war ein Joch Acker annähernd zwei Preßburger Metzen groß, was bedeutet, dass zu seinem Besäen zwei Metzen Saatgetreide gerechnet wurden (1 Metzen wog etwa $50 \mathrm{~kg}$ ).

Die Stellung und Zuordnung der Untertanen richteten sich nach der jeweiligen Besitzgröße, die in Lehen ausgewiesen wurde. Ursprünglich waren die Ganzlehen in der angegebenen Größe (Joch, später Preßburger Metzen) weit verbreitet. In der Herrschaft Landsee-Lackenbach etwa galt das Viertellehen als durchschnittliche Besitzgröße im 17. Jahrhundert, in der Maria Theresianischen Zeit wetteiferten die Achtellehen infolge Besitzteilungen mit den Viertellehen; Ganzlehen waren selten. Die Wiesen wurden, wie erwähnt, nach Tagwerken berechnet; wie etwa der Preßburger Metzen war z.B. eine bestimmte Wiese 2 Tagwerk groß, was somit jener Fläche entsprach, die man in zwei Tagen abmähen konnte. Ein eigenes Kapitel stellten die Weingärten und die mit diesen verbundenen Rechte und Pflichten dar. Für die Untertanengemeinden war die Weinausschank von Bedeutung, die den Untertanen zwischen Michaeli (29. September) und Weihnachten erlaubt war.

Die bäuerliche Wirtschaft bestand aus den Hofgründen (Haus, Hof, Stadel bzw. Garten) und Überlandgründen. Die rechtlich-soziale Stellung richtete sich danach, ob ein Untertan über Haus, Hof und Lehensgründe verfügte, oder aber nur ein Haus innehatte bzw. es nur bewohnte. So gliederten sich die Ortsbewohner auf Lehensbauer (ausgestattet mit 1 Ganzlehen, Halb-, Viertel- oder Achtelehen), Söllner (zsellér, inquilinus) mit bzw. ohne Haus (Inwohner, subinquilinus). In den elf Komitaten Transdanubiens zeigen folgende Daten die Relationen zwischen den einzelnen Gruppen:

\begin{tabular}{lrrrrrrr}
\hline \multirow{2}{*}{ Komitat } & \multicolumn{2}{c}{ Lehensbauern } & \multicolumn{2}{c}{ Söllner mit Haus } & \multicolumn{2}{c}{ Söllner ohne Haus } & \multirow{2}{*}{ insgesamt } \\
\cline { 2 - 6 } & absolut & relativ & absolut & relativ & absolut & relativ & \\
\hline Baranya & 13.942 & 82,99 & 2.539 & 15,11 & 319 & 1,90 & 16.800 \\
Fejér & 5.380 & 76,94 & 1.229 & 17,57 & 384 & 5,49 & 6.993 \\
Györ & 2.107 & 37,96 & 2.624 & 47,28 & 819 & 14,76 & 5.550 \\
Komárom & 3.749 & 66,08 & 1.547 & 27,27 & 377 & 6,65 & 5.673 \\
Moson & 4.113 & 55,75 & 1.442 & 19,55 & 1.822 & 24,70 & 7.377 \\
Somogy & 10.501 & 76,79 & 2.415 & 17,66 & 759 & 5,55 & 13.675 \\
Sopron & 11.029 & 58,87 & 4.633 & 24,73 & 3.072 & 16,40 & 18,734 \\
Tolna & 8.912 & 67,57 & 3.086 & 23,40 & 1.191 & 9,03 & 13.189 \\
Vas & 16.318 & 72,39 & 4.586 & 20,34 & 1.638 & 7,27 & 22.542 \\
Veszprém & 4.942 & 58,63 & 2.706 & 32,10 & 781 & 9,27 & 8.429 \\
Zala & 9.924 & 50,48 & 8.923 & 45,39 & 812 & 4,13 & 19.659 \\
\hline
\end{tabular}

Die Tabelle veranschaulicht die wirtschaftlich-soziale Gliederung der Untertanen. ${ }^{5}$ Zweifelsohne waren die Lehensbauern besser ausgestattet als die Söllner mit oder gar ohne Haus. Diesbezüglich nahm das Komitat Baranya mit 82,99\% 
die erste, mit 37,96\% das Komitat Györ die letzte Stelle ein. Umgekehrt führten die Söllner mit Haus mit 47,28\% im Komitat Györ, den niedrigsten Anteil zeigte mit $15,11 \%$ das Komitat Baranya. Die Söllner ohne Haus kletterten im Komitat Sopron auf 16,40\%, in Moson sogar auf 24,70\%. Ohne hier zu sehr ins Detail zu gehen, lässt sich jedenfalls feststellen, dass die Söllner, vor allem jene ohne Haus, mit der grundherrschaftlichen Eigenwirtschaft in Zusammenhang gebracht werden können. Vermutlich lebten viele von ihnen vom Handwerk.

Nun wäre es wichtig, die unter Eid abgelegten Bekenntnisse näher zu betrachten. Wie schon angedeutet, war es Sinn und Zweck des Urbarialpatentes, die Abgaben und Leistungen der grundherrschaftlichen Untertanen neu zu regeln, besser gesagt: rationell zu gestalten und in einheitliche Bahnen zu lenken. Dazu dienten die Vorerhebungen oder Befragungen, denn ohne Vorkenntnisse über die tatsächliche Lage wäre es kaum möglich gewesen, Ordnung zu schaffen und ein neues System einzuführen. Zum besseren Verständnis mögen die Unterlagen über das Komitat Sopron/Ödenburg vor Augen geführt werden. Die bereits zitierten zwei Bände liefern ein verblüffendes Bild über die Lage der herrschaftlichen Untertanen. Die Urbare wurden seinerzeit mit dem Zweck angelegt, die zu erwartenden Einnahmen der Grundherrschaft bzw. umgekehrt die Abgaben und Leistungen der Untertanen aufzuzeichnen. Die Grundherren konnten sich daran halten, die Untertanen aber mussten sich nach diesen Vorschriften richten. Die Urbare waren folglich im rechtlichen und wirtschaftlichen Sinne die wichtigste Rechtsgrundlage in den Beziehungen zwischen dem Grundherrn und seinen Untertanen. Die wie gesagt in zwei Bänden veröffentlichten Materialien erstrecken sich unter Berücksichtigung der jeweiligen Protokollsprache auf das Gebiet des heutigen Burgenlandes und den östlichen Teil des Ödenburger Komitats. Durch die dokumentierten Fakten werden deutliche Unterschiede zwischen den zwei Teilen erkennbar. Gleich unter Punkt 1 finden sich die Antworten auf die Frage, ob für die befragte Ortschaft ein Urbar aufliegt oder nicht. Bei den 98 Gemeinden im östlichen Teil gab es lediglich in 31 Fällen Urbare oder zumindest einen Kontrakt, 67 Gemeinden, also zwei Drittel, verfügten offenbar nicht darüber: entweder hatten die Auskunft gebenden Untertanen die sie betreffenden Urbare oder Kontrakte nicht zu Gesicht bekommen und wussten kaum etwas darüber, oder aber - wie es auch hieß - waren diese hochwichtigen Dokumente in den Kriegswirren verloren gegangen, in welchem Zusammenhang der Kuruzzenaufstand (1703-1711) wiederholt erwähnt wird. Im westlichen Teil des Komitates herrschten noch prekärere Zustände: Von den 108 Gemeinden behaupteten 94, sie besäßen kein Urbar, oder aber war ihnen dieses nicht bekannt. Unter den 31 Fällen im östlichen und bei 14 im westlichen Teil gab es Kontrakte zwischen dem Grundherrn und der Untertanengemeinde bzw. wurden in den Jahrzehnten vor der Maria Theresianischen Urbarialverordnung vor dem grundherrschaftlichen Gericht (úriszék) die Vorstellungen des Grundherrn bekannt gemacht - gleichviel, ob die Untertanen 
damit einverstanden waren oder nicht. Wo man sich auf kein Urbar stützen konnte, wurden die Abgaben und Leistungen nach altem Gebrauch verlangt bzw. eingefordert, gegen diese Praxis gab es bis dahin kein Rechtsmittel.

Man kann nicht umhin, in diesem Zusammenhang auch einen Blick auf die Verfassung der Untertanensiedlungen zu werfen. Auch diesbezüglich gab es Unterschiede zwischen dem östlichen (ungarischen) und dem westlichen (deutschen) Teil des Komitates: Von den 98 Orten im Osten waren 75 voll besiedelt, aber auch bei den restlichen 23 überstieg die Menge an leeren Häuser - außer in Csepreg mit 67 - in keinem Ort die Zahl Fünf. Im burgenländisch-westungarischen Teil ergaben die 65 voll besiedelten Orte 60,18\% der Gesamtzahl. Auch die Zahl der öden Lehen (Hofstätten bzw. Häuser) erreichte in den restlichen 48 Ortschaften 20 (Nikitsch), 22 (Draßmarkt), 28 (Weingraben) bzw. sogar 44 (Kaisersdorf). Neben der Armut der Einwohner, bedingt durch schlechte Bodenqualität und Witterungsverhältnisse (Regengüsse, Überschwemmungen) wird in einigen Orten sogar auf die Kuruzzenrummel als Ursache für die Wüstungen verwiesen.

Allein die hier angeführten Beispiele belehren darüber, wie akut nötig es war, mit Reformmaßnahmen in das Leben am Lande einzugreifen. In Fortsetzung der angeführten Argumente soll nun aufgezeichnet werden, was die bäuerlichen Untertanen dem Grundherrn gegenüber ,schuldig waren“. Vorrangig ist der Zins oder das Rauchgeld zu nennen, das nach einem Ganzlehen auf $1 \mathrm{fl}$ berechnet wurde. Die Abgaben erfolgten zumeist in Naturalien, wobei hier der Zehent oder das Neuntel zu nennen sind. Letzterer war je nach Größe des Lehens und zwar in Getreide (Roggen, Weizen, Gerste, Hafer) zu leisten, selbstverständlich war dafür nicht nur die Größe des Hofes, sondern de facto die jährliche Ernte ausschlaggebend. Die Bemessung der Abgaben erstreckte sich aber auf alle Produkte, die die bäuerliche Wirtschaft erzeugte. Wiederum nach einem Ganzlehen berechnet, mussten jährlich 2 Hühner, 2 Kapaune und 12 Eier, ferner eine halbe Butter für die Herrschaftsküche abgeliefert werden, es kamen aber auch Lamm und Bienenstock, d.h. Honig dazu. Diese konnten auch in Geld (4 bzw. 6 Kreuzer) abgelöst werden. Je 30 Untertanen waren verpflichtet, ein Kalb oder stattdessen 30 Groschen zu zahlen. Gleichviel, ob es in einem Ort Weingärten gab oder nicht, waren alle Untertanen vom Bergrecht betroffen. Wenn ja, mussten sie auch von ihrem Wein das Neuntel leisten, andernfalls mussten sie es in Geld ablösen.

Als schwere Belastung kann der Robot genannt werden, dem der bäuerliche Untertan in Form von Handrobot und Zugrobot nachzukommen hatte. Der Zugrobot betrug mit Pferden oder Ochsen jährlich 52, der Handrobot 104 Tage nach einem Ganzlehen. Der Handrobot für Söllner mit Haus betrug jährlich 18 Tage, ohne Haus 12 Tage. Es war nicht einheitlich geregelt, ob der Hin- und Rückweg in die Arbeitszeit inbegriffen waren. Diese war theoretisch wöchentlich zu verrichten; wenn ein Bauer nicht in der Lage war, dem Zugrobot nachzukommen, musste er dafür jeweils mit zwei Tagen Handrobot aufkommen. Ein permanentes 
Problem war es, wenn der Grundherr den Robot drosselte und sie dann zu den „Stoßzeiten“ verlangte, auch wenn die Bauern zugleich ihren eigenen Arbeiten nachkommen mussten. Je vier Ganzlehen oder Sessionen waren zu einer langen Fuhr mit einem Vierspann verpflichtet, diese durfte allerdings zwei Tagreisen nicht überschreiten; bei Schlechtwetter und unzureichenden Straßenverhältnissen mussten die Bauern aber dieser Pflicht nicht nachkommen.

Die Jagd war den bäuerlichen Untertanen untersagt, doch es war Pflicht, ihren Grundherrn jährlich drei Tage auf der Jagd zu begleiten. Es war ihnen aber gestattet, ihren Bedarf an Holz aus den Waldungen zu decken.

Der Grundherr bestimmte das tägliche Leben der Untertanen auch in dem Sinne, dass der Richter nach seinem Vorschlag gewählt wurde. Eine gewisse Lockerung war es, wenn der Grundherr drei Kandidaten für das Richteramt vorschlug; die Untertanen konnten sich dann für einen dieser drei entscheiden. Die Geschworenen durften sie selbst wählen. Den Dorfnotar stellte in den meisten Fällen der Schulmeister, wenn es im Ort eine Schule gab.

Ein eigenes Kapitel stellte die Freizügigkeit dar. Ohne Erlaubnis des Grundherrn durften die Untertanen selbst im eigenen Herrschaftsgebiet den Wohnort nicht wechseln. Wenn es ihnen aber gestattet wurde, mussten sie dafür das Abzugsgeld zahlen. Ihre Mobilien durften sie mitführen; an Grund und Boden hatten sie lediglich Nutzungsrecht, ihr Haus durften sie allerdings veräußern.

All die Rechten und Pflichten, Abgaben und Leistungen waren in den Urbaren schriftlich festgehalten. Wie bereits gezeigt wurde, gab es aber genug Fälle ohne Urbar bzw. Kontrakt, für Missbräuche und Übergriffe waren folglich Tür und Tor geöffnet. Welche Beweggründe Maria Theresia auch dafür gehabt haben mag, in die Verhältnisse zwischen Herrn und Untertan einzugreifen, sie ebnete damit den Weg für die spätere Bauernbefreiung. Allein schon von dieser Warte aus bleiben ihre Bedeutung und ihre Leistungen unvergänglich. In der Kontroverse zwischen den imperialen und nationalen Interessen fällt es seitens Ungarn nicht leicht, die Verdienste Maria Theresias vorurteilsfrei anzuerkennen. Sicherlich wirft es ein schiefes Licht auf die ungarische Adelspolitik, dass sich nicht die Landesherren, sondern eine Habsburgerin die Verbesserung der Lage der bäuerlichen Untertanen zur eigenen Sache machte. Dazu war ihre diesbezügliche Politik völlig konform mit den Interessen des Habsburgerreiches. Diese Feststellung will jedoch niemanden dazu verleiten, besondere Sympathie für die Habsburger zu wecken. Es dient nur der Sachlichkeit und Unparteilichkeit, wenn eine klare Sicht der Dinge historische Fakten weder imperial, noch national gefärbt, sondern unvoreingenommen an ihre richtige Stelle rückt. Dazu lassen wir die Protokolle direkt sprechen: Was alles wurde aufgedeckt, um dann durch Gerechtigkeit oder Kompensation die Lage der bäuerlichen Bevölkerung Zukunft weisend zu verbessern?

Das wichtigste Resultat der Urbarialverordnung war aufgrund der Befragungen und ausgewerteten Antworten die Anlegung einer neuen, nach einheitlichen 
Kriterien festgelegten Regelung. Das umfangreiche Material ermöglicht im hier gegebenen Rahmen kaum eine eingehende Auseinandersetzung. In der Erwägung, welche Vorgangsweise besser wäre, empfiehlt es sich, das Problem anhand eines konkreten Beispiels unter die Lupe zu nehmen. Zu diesem Zweck wird hier das Urbarium des zur fürstlich Esterhazischen Herrschaft gehörigen Marktes Landsee mit Blumau herausgegriffen. ${ }^{6}$ (Uriginal Urbarien und Berg Bücher der Herrschaft Lackenbach vom Jahr 1767, auf Landsee bezogen lautet der Titel: (Urbarium des Marktes Lansee mit Blumau zu der fürstl. Esterhazischen Herrschaft Lansee gehörig. Burg Forchtenstein, Prot. 717)

Die ausführliche Beschreibung beinhaltet die Antworten auf die neun Fragen, die folgendermaßen betitelt sind:

Punkt 1. Von Bestimmung der Ansäßigkeiten

Punkt 2.Von denen Benefizien deren Unterthanen

Punkt 3. Von denen Robathen und Diensten deren Unterthanen

Punkt 4.Von den Gaben und übrigen Anlagen

Punkt 5. Von dem Neuntl, und dem Bergrecht

Punkt 6. Von denen Rechten vnd Gerechtsamen der Grundherren

Punkt 7. Von denen aufgehobenen und hinkünftig zu vermeidenden Mißbräuchen und Exzessen

Punkt 8. Von jenen so dem Unterthan verboten ist, und von denen hierauf gesetzten Strafen

Punkt 9. Von jenem, so zur innerlichen Einrichtung gehöret.

Ignatius Nagy Comitatus Soproniensis Assessor 1.s.

Stephanus Rohrer ejusdem S. Comitatus Jurassor 1.s. Urbarium

Durch die zwei Vertreter des Komitates wurde der Akt beglaubigt.

Es folgt - wie bei anderen Orten - die Urbarial-Tabelle, in der die Höfler, dann die Söllner mit, schließlich ohne Haus, namentlich eingetragen sind. In 16 Rubriken finden sich folgende Daten:

Eigenschaft der Ansässigkeit

Bestimmung der Ansässigkeit: Innerer, Äußerer Grund - Preßburger Metzen Joch Äcker - Tagwerk Wiesen

Jährliche Robathen: Zugrobath mit vier Stück Vieh - oder statt derselben Handrobath

Neuntl: aequivalent an barem - Geld

Zins und Gaben: Zins - Brennholz - Gespunnst - Schmalz - Kapaunen - Hünel - Eyer.

Die 32 Lehen der Güterklasse IV waren durchwegs $1 / 8$ groß, berechnet auf 1 oder 6/8 Preßburger Metzen bzw, 4 6/8 Joch Acker und 6/8 Tagwerk Wiesen; jährlich zw. 10-12 Tagen Zugrobot, 20-22 Tagen Handrobot; das Neuntel wurde 
zwischen 75-80 Denar in Geld abgelöst; der Zins betrug einheitlich 1 fl.; Brennholz 1/8 Klafter, Gespunnst 1 lb., Schmalz 0; Kapaune bzw. Hühner je 1 mit einzelnen Ausnahmen, Eier 5-11. Bei den 39 Söllnern mit Haus sind lediglich 18 Tage Handrobat und 1 fl Zins eingetragen, die 6 Söllner ohne Haus waren zu 12 Tagen Handrobot verpflichtet.

Wie bei ähnlicher Bodenqualität bestand auch in Landsee ein Ganzlehen aus 22 Joch Acker, jedes Joch wurde auf 2 Preßburger Metzen berechnet, dazu gehörten 6 Tagwerk Wiesen.

Die Verrichtung der Robot ist klar und deutlich formuliert: Ein ganzer Ansässiger ist schuldig, jede Woche einen Tag von Sonnenaufgang bis Sonnenuntergang mit Inbegriff des Hingangs und Zuruckgangs, mit Ausnahme jedoch deren zur Fütterung und zur Tränkung des Zugviehs erforderlichen Stunden mit zwei Stück Vieh, in Brache aber mit vier Zugvieh und ebenfalls mit eigenem Wagen, Pflug und Eggen nach altem Brauch dieses Ortes zu dienen. Im Weiteren wird penibel darauf geachtet, dass keine Missverständnisse aufkommen. Immerhin wird auch festgehalten: Wenn bezüglich der Verrichtung der Robot zwischen dem Grundherrn und dem Untertan Streit entstünde, kann sich letzterer an die Komitatsinstanzen wenden, dort wird nach Maß und Billigkeit darüber entschieden. (Punkt 3, § I)

Auch das bereits erwähnte Fuhrwerk wird hier genau beschrieben. Es ging um eine vierspannige Fuhr, zu der die Untertanen einmal jährlich angehalten wurden. Die Entfernungen mögen zwei Tage nicht überschreiten. Die weite oder vierspannige Fuhr darf allenfalls zur Zeit der Ernte und Weinlese einschließlich des Heumachens nicht angefordert werden. Sie darf weder auf das folgende Jahr verschoben, noch in Feld- oder andere Arbeit umgewandelt werden. Konnten die zwei Tage nicht eingehalten werden, musste für die Unkosten (Beherbergung, Verpflegung) der Grundherr aufkommen. Es wurde eingeführt, dass Maut und Dreißigst den Grundherrn belasteten. (Punkt 3, § XII)

Wie schon angemerkt, durfte sich der bäuerliche Untertan mit seinen Beschwerden künftighin an die Instanzen des Komitats wenden. Abgesehen von der einheitlichen Regelung mussten bei Kontraktverhandlungen zwischen dem Grundherr und der Untertanengemeinde Vertreter des Komitats anwesend sein, was auch ein Novum war. Der 7. Punkt des Urbars beinhaltet eine ganze Reihe von Neuigkeiten, die den Untertanen zugute kamen. Bemerkenswert lautet schon der einleitende Satz dazu:

Da dem Grundherrn als Beschützer deren Waisen die Obsorge über das Vermögen deren durch seine Unterthanen hinterlassenen Pupillen ohnehin oblieget... werden alle Verlassenschafts- oder Inventars- und Theilungs=Taxen von dem Vermögen des Unterthans, wie selbe immer Namen haben mögen vollständig aufgehoben. (Punkt 7, § 1)

Die Benefizien betreffend hatten die Untertanen u.a. zwischen Michaeli und Weihnachten freien Weinausschank. 
Die Untertanen durften künftighin ihre Waren frei verkaufen - die Benützung der herrschaftlichen Mühlen ist nicht mehr zwingend. Anachronistisch liest sich die Stelle über die Lieferung des eigenen Dungs auf die Herrschaftsfelder - ausgenommen die Weingärten - die nun abgestellt wurde. Auch die Entrichtung des Taglohns der herrschaftlichen Trabanten wird seitens der Untertanen abgeschafft. Die Untertanen sollen zum Kauf von Naturalien und Viktualien, insbesondere verdorbenem Wein und Brantwein nicht angehalten werden. Bäuerliche Gründe dürfen von der Grundherrschaft nur gegen Kompensation von gleicher Größe, Güter und Fruchtbarkeit eingezogen bzw. umgetauscht werden. Widrigenfalls sollen solche Gründe wieder zurückerstattet werden.

Waldnutzung: Dem Untertan ist gestattet, von dem windfällig- oder andern duerren Holz lediglich zu seiner eigenen Nothdurft sein Brennholz zu sammeln. Das erforderliche Bauholz ist dem Untertan aus dem eigenen Ortsgebiet durch die Erlaubnis der Grundherrschaft kostenlos zugewiesen, die Bäume dazu werden von der Grundherrschaft gekennzeichnet. (Punkt 1, §§ IV, V)

Unabhängig von bestimmten Lockerungen galt nach wie vor das Jagdverbot, speziell durfte kein Untertan Gewehr oder Jagdhunde halten. (Punkt 8, § XII) Dafür waren die Untertanen zur dreitägigen Jagd zwecks Ausrottung der wilden Tiere verpflichtet, Pulver und Blei erhielten sie von der Grundherrschaft. Diese Pflicht konnte weder durch Geld abgelöst, noch in eine andere Form von Robot umgetauscht werden. (Punkt $3, \S \mathrm{XVI}$ )

Wer aus eigenem Verschuldem seiner Robotpflicht nicht nachkommt, solle mit zwölf Prügeln bestraft werden. (Punkt $8, \S$ XIV) Die Bestrafung der Übeltäter wurde vor allem dahingehend gemildert, dass bei kleineren Delikten die Leibeszüchtigung nach Möglichkeit z.B. in zwei oder dreitägige Handarbeit umgewandelt wurde. Obwohl die Züchtigung durch Geldleistung oder Leibesstrafe nicht erlaubt sein sollte, konnte die letztere in bestimmten Fällen doch verwendet werden, der Strafmäßige sollte aber zuerst dahingehend geprüft werden, ob der/ die Betreffende eine gesunde und starke Person war. Wenn ja, sollte er nach Maß des Verbrechens höchstens mit 24 Stockstreichen, oder nach Art des Geschlechts mit 24 Karbatschstreichen gezüchtiget werden. Bei höherem Alter oder schwacher Statur kam der Straffällige höchstens auf drei Tage bei Brot und Wasser ins Gefängnis. (Punkt 8 , §§ III, IV) Selbstverständlich betrafen diese Bestrafungen kleinere Delikte der niederen (herrschaftlichen) Gerichtsbarkeit.

Die Maria Theresianische Urbarialverordnung führte im Wesentlichen, vornehmlich in Hinblick auf die Abgaben und Leistungen keine grundlegenden Änderungen ein, schuf aber in den mitunter unübersichtlichen Zuständen Ordnung und führte landesweit ein einheitliches System ein. Neben etlichen Erleichterungen waren dies Schritte, die den Weg in die Zukunft wiesen. 


\section{Anmerkungen}

1 Martin Mutschlechner, Maria Theresia 1717-1780. Strategin, Mutter, Reformerin, Wien 2017.

2 Vgl. die Eckdaten dazu: Kálmán Benda (Red.), Magyarország történeti kronológiája II. 15261848, [Historische Chronologie Ungarns II. 1526-1848], Budapest 1982, 575-587.

3 Ibolya Felhő (red.), Az úrbéres viszonyok Magyarországon Mária Terézia korában I. kötet Dunántúl, Budapest, 1970 [Die urbarialen Besitzverhältnisse in Ungarn zur Zeit Maria Theresias]. Bd. I., Budapest 1970, 11.

4 Peter Tóth, A Mária Terézia-kori úrbérrendezés kilenc kérdöpontos vizsgálatai Sopron vármegyében, I. Magyar és latin nyelvü vallomások (1767) Antworten auf die Neun Fragepunkte der Maria Theresianischen Urbarialregulierung im Komitat Sopron/Ödenburg, Sopron 1998 - József Tirnitz, Antworten auf die Neun Fragepunkte der Maria Theresianischen Urbarialregulierung im Komitat Sopron/Ödenburg, II. Deutsche Bekenntnisse (1767) A Mária Terézia-kori úrbérrendezés kilenc kérdöpontos vizsgálatai Sopron vármegyében, II. Német nyelvü vallomások (1767), Sopron 1999.

5 Anm. 3, Tabelle 8, 44.

6 Original Urbarien und Berg Bücher der Herrschaft Lackenbach vom Jahr 1767, auf Landsee bezogen lautet der Titel: Urbarium des Marktes Lansee mit Blumau zu der fürstl. Esterhazischen Herrschaft Lansee gehörig. Esterházy-Archiv Burg Forchtenstein, Prot. 717. 